\title{
Existence and Nonexistence of Positive Solutions for Coupled Riemann-Liouville Fractional Boundary Value Problems
}

\author{
Johnny Henderson, ${ }^{1}$ Rodica Luca, ${ }^{2}$ and Alexandru Tudorache ${ }^{3}$ \\ ${ }^{1}$ Department of Mathematics, Baylor University, Waco, TX 76798-7328, USA \\ ${ }^{2}$ Department of Mathematics, Gh. Asachi Technical University, 700506 Iasi, Romania \\ ${ }^{3}$ Faculty of Computer Engineering and Automatic Control, Gh. Asachi Technical University, 700050 Iasi, Romania
}

Correspondence should be addressed to Rodica Luca; rlucatudor@yahoo.com

Received 12 January 2016; Revised 13 April 2016; Accepted 14 June 2016

Academic Editor: Josef Diblík

Copyright (C) 2016 Johnny Henderson et al. This is an open access article distributed under the Creative Commons Attribution License, which permits unrestricted use, distribution, and reproduction in any medium, provided the original work is properly cited.

We investigate the existence and nonexistence of positive solutions for a system of nonlinear Riemann-Liouville fractional differential equations with two parameters, subject to coupled integral boundary conditions.

\section{Introduction}

Fractional differential equations describe many phenomena in various fields of engineering and scientific disciplines such as physics, biophysics, chemistry, biology, economics, control theory, signal and image processing, aerodynamics, viscoelasticity, and electromagnetics (see [1-5]). Integral boundary conditions arise in thermal conduction problems, semiconductor problems, and hydrodynamic problems (see, e.g., [6-9]).

We consider the system of nonlinear fractional differential equations with parameters

$$
\begin{aligned}
& D_{0+}^{\alpha} u(t)+\lambda f(t, u(t), v(t))=0, \quad t \in(0,1), \\
& D_{0+}^{\beta} v(t)+\mu g(t, u(t), v(t))=0, \quad t \in(0,1),
\end{aligned}
$$

with the coupled integral boundary conditions

$$
\begin{aligned}
u(0) & =u^{\prime}(0)=\cdots=u^{(n-2)}(0)=0, \\
u^{\prime}(1) & =\int_{0}^{1} v(s) d H(s), \\
v(0) & =v^{\prime}(0)=\cdots=v^{(m-2)}(0)=0, \\
v^{\prime}(1) & =\int_{0}^{1} u(s) d K(s),
\end{aligned}
$$

where $\alpha \in(n-1, n], \beta \in(m-1, m], n, m \in \mathbb{N}, n, m \geq 3$, $D_{0+}^{\alpha}$ and $D_{0+}^{\beta}$ denote the Riemann-Liouville derivatives of orders $\alpha$ and $\beta$, respectively, and the integrals from $(B C)$ are Riemann-Stieltjes integrals. The boundary conditions $(B C)$ include multipoint and integral boundary conditions, as well as the sum of these in a single framework.

Under some assumptions on the nonnegative functions $f$ and $g$, we present intervals for the parameters $\lambda$ and $\mu$ such that positive solutions of $(S)-(B C)$ exist. By a positive solution of problem $(S)-(B C)$ we mean a pair of functions $(u, v) \in C\left([0,1] ; \mathbb{R}_{+}\right) \times C\left([0,1] ; \mathbb{R}_{+}\right)$satisfying $(S)$ and $(B C)$ with $u(t)>0$ for all $t \in(0,1]$ or $v(t)>0$ for all $t \in(0,1]$. The nonexistence of positive solutions for $(S)-(B C)$ is also investigated. The existence, multiplicity, and nonexistence of positive solutions $(u(t), v(t) \geq 0$ for all $t \in[0,1]$ and $(u, v) \neq(0,0))$ for system $(S)$ with different coupled boundary conditions, namely,

$$
\begin{aligned}
& u(0)=u^{\prime}(0)=\cdots=u^{(n-2)}(0)=0, \\
& u(1)=\int_{0}^{1} v(s) d H(s), \\
& v(0)=v^{\prime}(0)=\cdots=v^{(m-2)}(0)=0, \\
& v(1)=\int_{0}^{1} u(s) d K(s),
\end{aligned}
$$


were investigated in $[10,11]$ (where $f$ and $g$ are nonnegative and nonsingular functions) and in [12] (where $\lambda=\mu=1$ and $f(t, u, v)$ and $g(t, u, v)$ are replaced by $\tilde{f}(t, v)$ and $\tilde{g}(t, u)$, respectively, with $\widetilde{f}$ and $\widetilde{g}$ nonnegative functions, singular or not). In this paper, Green's functions associated with problem $(S)-(B C)$, the inequalities satisfied by these functions, and the cone defined in the proof of the main results are different than the corresponding ones that the authors used in $[10$ 12] for problem $(S)-\left(B C_{1}\right)$. Existence results for the positive solutions of problem $(S)-(B C)$, where $f$ and $g$ are signchanging functions which may be singular at $t=0$ or $t=1$ and satisfy some different assumptions than those used in this paper, were obtained in [13]. We also mention the paper [14], where the authors studied the existence and multiplicity of positive solutions for system (S) with $\alpha=\beta, \lambda=\mu$ and the boundary conditions $u^{(i)}(0)=v^{(i)}(0)=0, i=0, \ldots, n-2$, $u(1)=a v(\xi)$, and $v(1)=b u(\eta)$, with $\xi, \eta \in(0,1), 0<$ $a b \xi \eta<1$, and $f$ and $g$ are sign-changing nonsingular or singular functions. The results obtained in [14] are relying on a nonlinear alternative of Leray-Schauder type and the Krasnosel'skii's fixed point theorem. For other recent results concerning the coupled fractional boundary value problems we refer the reader to [15-17].

The paper is organized as follows. Section 2 contains some auxiliary results which investigate a nonlocal boundary value problem for fractional differential equations and presents the properties of Green's functions associated to our problem $(S)$ $(B C)$. In Section 3, we prove the main existence theorems for the positive solutions with respect to a cone for $(S)$ $(B C)$ which are based on the Guo-Krasnosel'skii fixed point theorem, and then the nonexistence of positive solutions is studied in Section 4. Finally, in Section 5, two examples are given to illustrate our main results.

\section{Auxiliary Results}

In this section, we present some auxiliary results that will be used to prove our main results.

We consider the fractional differential system

$$
\begin{aligned}
& D_{0+}^{\alpha} u(t)+x(t)=0, \quad t \in(0,1), \\
& D_{0+}^{\beta} v(t)+y(t)=0, \quad t \in(0,1),
\end{aligned}
$$

with the coupled integral boundary conditions

$$
\begin{aligned}
& u(0)=u^{\prime}(0)=\cdots=u^{(n-2)}(0)=0, \\
& u^{\prime}(1)=\int_{0}^{1} v(s) d H(s), \\
& v(0)=v^{\prime}(0)=\cdots=v^{(m-2)}(0)=0, \\
& v^{\prime}(1)=\int_{0}^{1} u(s) d K(s),
\end{aligned}
$$

where $\alpha \in(n-1, n], \beta \in(m-1, m], n, m \in \mathbb{N}, n, m \geq 3$, and $H, K:[0,1] \rightarrow \mathbb{R}$ are functions of bounded variation.
Lemma 1 (see [13]). If $H, K:[0,1] \rightarrow \mathbb{R}$ are functions of bounded variation, $\Delta=(\alpha-1)(\beta-1)-\left(\int_{0}^{1} \tau^{\alpha-1} d K(\tau)\right)$ $\left(\int_{0}^{1} \tau^{\beta-1} d H(\tau)\right) \neq 0$ and $x, y \in C(0,1) \cap L^{1}(0,1)$, then the pair of functions $(u, v) \in C([0,1]) \times C([0,1])$ given by

$$
\begin{array}{r}
u(t)=\int_{0}^{1} G_{1}(t, s) x(s) d s+\int_{0}^{1} G_{2}(t, s) y(s) d s, \\
t \in[0,1], \\
v(t)=\int_{0}^{1} G_{3}(t, s) y(s) d s+\int_{0}^{1} G_{4}(t, s) x(s) d s, \\
t \in[0,1],
\end{array}
$$

is solution of problem (1)-(2), where

$$
\begin{aligned}
& G_{1}(t, s)=g_{1}(t, s)+\frac{t^{\alpha-1}}{\Delta}\left(\int_{0}^{1} \tau^{\beta-1} d H(\tau)\right) \\
& \cdot\left(\int_{0}^{1} g_{1}(\tau, s) d K(\tau)\right), \quad \forall t, s \in[0,1], \\
& G_{2}(t, s)=\frac{(\beta-1) t^{\alpha-1}}{\Delta} \int_{0}^{1} g_{2}(\tau, s) d H(\tau), \\
& G_{3}(t, s)=g_{2}(t, s)+\frac{t^{\beta-1}}{\Delta}\left(\int_{0}^{1} \tau^{\alpha-1} d K(\tau)\right) \\
& \cdot\left(\int_{0}^{1} g_{2}(\tau, s) d H(\tau)\right), \quad \forall t, s \in[0,1], \\
& G_{4}(t, s)=\frac{(\alpha-1) t^{\beta-1}}{\Delta} \int_{0}^{1} g_{1}(\tau, s) d K(\tau),
\end{aligned}
$$

$$
\forall t, s \in[0,1]
$$

$$
\begin{aligned}
& g_{1}(t, s)=\frac{1}{\Gamma(\alpha)} \\
& \cdot \begin{cases}t^{\alpha-1}(1-s)^{\alpha-2}-(t-s)^{\alpha-1}, & 0 \leq s \leq t \leq 1, \\
t^{\alpha-1}(1-s)^{\alpha-2}, & 0 \leq t \leq s \leq 1,\end{cases} \\
& g_{2}(t, s)=\frac{1}{\Gamma(\beta)} \\
& \qquad \begin{cases}t^{\beta-1}(1-s)^{\beta-2}-(t-s)^{\beta-1}, & 0 \leq s \leq t \leq 1, \\
t^{\beta-1}(1-s)^{\beta-2}, & 0 \leq t \leq s \leq 1 .\end{cases}
\end{aligned}
$$

Lemma 2 (see [13]). The functions $g_{1}, g_{2}$ given by (5) have the following properties:

(a) $g_{1}, g_{2}:[0,1] \times[0,1] \rightarrow \mathbb{R}_{+}$are continuous functions, and $g_{1}(t, s)>0, g_{2}(t, s)>0$ for all $(t, s) \in(0,1] \times(0,1)$.

(b) $g_{1}(t, s) \leq h_{1}(s), g_{2}(t, s) \leq h_{2}(s)$ for all $(t, s) \in$ $[0,1] \times[0,1]$, where $h_{1}(s)=s(1-s)^{\alpha-2} / \Gamma(\alpha)$ and $h_{2}(s)=$ $s(1-s)^{\beta-2} / \Gamma(\beta)$ for all $s \in[0,1]$.

(c) $g_{1}(t, s) \geq t^{\alpha-1} h_{1}(s), g_{2}(t, s) \geq t^{\beta-1} h_{2}(s)$ for all $(t, s) \in$ $[0,1] \times[0,1]$. 
Lemma 3 (see [13]). If $H, K:[0,1] \rightarrow \mathbb{R}$ are nondecreasing functions, and $\Delta>0$, then $G_{i},(i=1, \ldots, 4)$ given by (4) are continuous functions on $[0,1] \times[0,1]$ and satisfy $G_{i}(t, s) \geq 0$ for all $(t, s) \in[0,1] \times[0,1],(i=1, \ldots, 4)$. Moreover, if $x, y \in$ $C(0,1) \cap L^{1}(0,1)$ satisfy $x(t) \geq 0, y(t) \geq 0$ for all $t \in(0,1)$, then the solution $(u, v)$ of problem (1)-(2) given by (3) satisfies $u(t) \geq 0, v(t) \geq 0$ for all $t \in[0,1]$.

Lemma 4. Assume that $H, K:[0,1] \rightarrow \mathbb{R}$ are nondecreasing functions and $\Delta>0$. Then the functions $G_{i},(i=1, \ldots, 4)$ satisfy for all $(t, s) \in[0,1] \times[0,1]$ the following relations:

$$
\begin{aligned}
& \left(a_{1}\right) G_{1}(t, s) \leq J_{1}(s), \text { where } J_{1}(s)=h_{1}(s)+ \\
& (1 / \Delta)\left(\int_{0}^{1} \tau^{\beta-1} d H(\tau)\right)\left(\int_{0}^{1} g_{1}(\tau, s) d K(\tau)\right) . \\
& \left(a_{2}\right) G_{1}(t, s) \geq t^{\alpha-1} J_{1}(s) . \\
& \left(b_{1}\right) G_{2}(t, s) \leq \quad J_{2}(s) \text {, where } J_{2}(s)=((\beta- \\
& 1) / \Delta) \int_{0}^{1} g_{2}(\tau, s) d H(\tau) . \\
& \left(b_{2}\right) G_{2}(t, s)=t^{\alpha-1} J_{2}(s) . \\
& \left(c_{1}\right) G_{3}(t, s) \leq J_{3}(s), \text { where } J_{3}(s)=h_{2}(s)+ \\
& (1 / \Delta)\left(\int_{0}^{1} \tau^{\alpha-1} d K(\tau)\right)\left(\int_{0}^{1} g_{2}(\tau, s) d H(\tau)\right) . \\
& \left(c_{2}\right) G_{3}(t, s) \geq t^{\beta-1} J_{3}(s) . \\
& \left(d_{1}\right) G_{4}(t, s) \leq \quad J_{4}(s), \text { where } J_{4}(s)=((\alpha- \\
& 1) / \Delta) \int_{0}^{1} g_{1}(\tau, s) d K(\tau) . \\
& \left(d_{2}\right) G_{4}(t, s)=t^{\beta-1} J_{4}(s) .
\end{aligned}
$$

Proof. The above inequalities follow from the properties of the functions $g_{i},(i=1, \ldots, 4)$ from Lemma 2 .

Lemma 5. Assume that $H, K:[0,1] \rightarrow \mathbb{R}$ are nondecreasing functions, $\Delta>0$, and $x, y \in C(0,1) \cap L^{1}(0,1), x(t) \geq 0$, and $y(t) \geq 0$ for all $t \in(0,1)$. Then the solution $(u(t), v(t))$, $t \in[0,1]$ of problem (1)-(2) given by (3) satisfies the inequalities $u(t) \geq t^{\alpha-1} u\left(t^{\prime}\right), v(t) \geq t^{\beta-1} v\left(t^{\prime}\right)$, for all $t, t^{\prime} \in[0,1]$.

Proof. By using Lemma 4, we obtain for all $t, t^{\prime} \in[0,1]$ the following inequalities:

$$
\begin{aligned}
& u(t)=\int_{0}^{1} G_{1}(t, s) x(s) d s+\int_{0}^{1} G_{2}(t, s) y(s) d s \\
& \geq t^{\alpha-1}\left(\int_{0}^{1} J_{1}(s) x(s) d s+\int_{0}^{1} J_{2}(s) y(s) d s\right) \\
& \geq t^{\alpha-1}\left(\int_{0}^{1} G_{1}\left(t^{\prime}, s\right) x(s) d s+\int_{0}^{1} G_{2}\left(t^{\prime}, s\right) y(s) d s\right) \\
& =t^{\alpha-1} u\left(t^{\prime}\right), \\
& v(t)=\int_{0}^{1} G_{3}(t, s) y(s) d s+\int_{0}^{1} G_{4}(t, s) x(s) d s
\end{aligned}
$$

$$
\begin{aligned}
& \geq t^{\beta-1}\left(\int_{0}^{1} J_{3}(s) y(s) d s+\int_{0}^{1} J_{4}(s) x(s) d s\right) \\
& \geq t^{\beta-1}\left(\int_{0}^{1} G_{3}\left(t^{\prime}, s\right) y(s) d s+\int_{0}^{1} G_{4}\left(t^{\prime}, s\right) x(s) d s\right) \\
& =t^{\beta-1} v\left(t^{\prime}\right) .
\end{aligned}
$$

In the proof of our main existence results we will use the Guo-Krasnosel'skii fixed point theorem presented below (see $[18])$.

Theorem 6. Let $X$ be a Banach space and let $C \subset X$ be a cone in X. Assume $\Omega_{1}$ and $\Omega_{2}$ are bounded open subsets of $X$ with $0 \in \Omega_{1} \subset \overline{\Omega_{1}} \subset \Omega_{2}$ and let $\left.\mathscr{A}: C \cap \overline{\left(\Omega_{2}\right.} \backslash \Omega_{1}\right) \rightarrow C$ be a completely continuous operator such that either

(i) $\|\mathscr{A} u\| \leq\|u\|, u \in C \cap \partial \Omega_{1}$, and $\|\mathscr{A} u\| \geq\|u\|, u \in$ $C \cap \partial \Omega_{2}$, or

(ii) $\|\mathscr{A} u\| \geq\|u\|, u \in C \cap \partial \Omega_{1}$, and $\|\mathscr{A} u\| \leq\|u\|, u \in$ $C \cap \partial \Omega_{2}$.

Then $\mathscr{A}$ has a fixed point in $C \cap\left(\overline{\Omega_{2}} \backslash \Omega_{1}\right)$.

\section{Existence Results for the Positive Solutions}

In this section, we will give sufficient conditions on $\lambda, \mu, f$, and $g$ such that positive solutions with respect to a cone for our problem $(S)-(B C)$ exist.

We present now the assumptions that we will use in the sequel:

(H1) $H, K:[0,1] \rightarrow \mathbb{R}$ are nondecreasing functions and $\Delta=(\alpha-1)(\beta-1)-\left(\int_{0}^{1} \tau^{\alpha-1} d K(\tau)\right) \times\left(\int_{0}^{1} \tau^{\beta-1} d H(\tau)\right)>$ 0.

(H2) The functions $f, g:[0,1] \times[0, \infty) \times[0, \infty) \rightarrow[0, \infty)$ are continuous.

For $c \in(0,1)$, we introduce the following extreme limits:

$$
\begin{aligned}
f_{0}^{s} & =\limsup _{u+v \rightarrow 0^{+}} \max _{t \in[0,1]} \frac{f(t, u, v)}{u+v}, \\
g_{0}^{s} & =\limsup _{u+v \rightarrow 0^{+}} \max _{t \in[0,1]} \frac{g(t, u, v)}{u+v}, \\
f_{0}^{i} & =\liminf _{u+v \rightarrow 0^{+}} \min _{t \in[c, 1]} \frac{f(t, u, v)}{u+v}, \\
g_{0}^{i} & =\liminf _{u+v \rightarrow 0^{+}} \min _{t \in[c, 1]} \frac{g(t, u, v)}{u+v}, \\
f_{\infty}^{s} & =\limsup _{u+v \rightarrow \infty} \max _{t \in[0,1]} \frac{f(t, u, v)}{u+v}, \\
g_{\infty}^{s} & =\limsup _{u+v \rightarrow \infty} \max _{t \in[0,1]} \frac{g(t, u, v)}{u+v},
\end{aligned}
$$




$$
\begin{aligned}
f_{\infty}^{i} & =\liminf _{u+v \rightarrow \infty} \min _{t \in[c, 1]} \frac{f(t, u, v)}{u+v}, \\
g_{\infty}^{i} & =\liminf _{u+v \rightarrow \infty} \min _{t \in[c, 1]} \frac{g(t, u, v)}{u+v} .
\end{aligned}
$$

In the definitions of the extreme limits above, the variables $u$ and $v$ are nonnegative.

By using Lemma 1, a solution of the following nonlinear system of integral equations

$$
\begin{aligned}
u(t)= & \lambda \int_{0}^{1} G_{1}(t, s) f(s, u(s), v(s)) d s \\
& +\mu \int_{0}^{1} G_{2}(t, s) g(s, u(s), v(s)) d s, \\
v(t)= & \mu \int_{0}^{1} G_{3}(t, s) g(s, u(s), v(s)) d s \quad \\
& +\lambda \int_{0}^{1} G_{4}(t, s) f(s, u(s), v(s)) d s,
\end{aligned}
$$

$t \in[0,1]$

is a solution for problem $(S)-(B C)$.

We consider the Banach space $X=C([0,1])$ with the supremum norm $\|\cdot\|$ and the Banach space $Y=X \times X$ with the norm $\|(u, v)\|_{Y}=\|u\|+\|v\|$. We define the cone $P \subset Y$ by

$$
\begin{aligned}
P & =\left\{(u, v) \in Y ; u(t) \geq t^{\alpha-1}\|u\|, v(t) \geq t^{\beta-1}\|v\|, \forall t\right. \\
& \in[0,1]\} .
\end{aligned}
$$

For $\lambda, \mu>0$, we introduce the operators $T_{1}, T_{2}: Y \rightarrow X$ and $\mathscr{T}: Y \rightarrow Y$ defined by

$$
\begin{aligned}
T_{1}(u, v)(t)= & \lambda \int_{0}^{1} G_{1}(t, s) f(s, u(s), v(s)) d s \\
& +\mu \int_{0}^{1} G_{2}(t, s) g(s, u(s), v(s)) d s, \\
\quad 0 \leq t \leq 1, & \\
T_{2}(u, v)(t)= & \mu \int_{0}^{1} G_{3}(t, s) g(s, u(s), v(s)) d s \\
& +\lambda \int_{0}^{1} G_{4}(t, s) f(s, u(s), v(s)) d s, \\
& 0 \leq t \leq 1,
\end{aligned}
$$

and $\mathscr{T}(u, v)=\left(T_{1}(u, v), T_{2}(u, v)\right),(u, v) \in Y$. It is clear that if $(u, v)$ is a fixed point of operator $\mathscr{T}$, then $(u, v)$ is a positive solution of problem $(S)-(B C)$.

Lemma 7. If (H1)-(H2) hold, then $\mathscr{T}: P \rightarrow P$ is a completely continuous operator.
Proof. Let $(u, v) \in P$ be an arbitrary element. Because $T_{1}(u, v)$ and $T_{2}(u, v)$ satisfy problem (1)-(2) for $x(t)=\lambda f(t, u(t), v(t))$, $t \in[0,1]$, and $y(t)=\mu g(t, u(t), v(t)), t \in[0,1]$, then by Lemma 5, we obtain

$$
\begin{aligned}
& T_{1}(u, v)(t) \geq t^{\alpha-1} T_{1}(u, v)\left(t^{\prime}\right), \\
& T_{2}(u, v)(t) \geq t^{\beta-1} T_{2}(u, v)\left(t^{\prime}\right),
\end{aligned}
$$

$$
\forall t, t^{\prime} \in[0,1],
$$

and so

$$
\begin{aligned}
& T_{1}(u, v)(t) \geq t^{\alpha-1}\left\|T_{1}(u, v)\right\|, \\
& T_{2}(u, v)(t) \geq t^{\beta-1}\left\|T_{2}(u, v)\right\|,
\end{aligned}
$$

$$
\forall t \in[0,1] .
$$

By (H2) and the above inequalities, we deduce that $\mathscr{T}(u, v) \in P$. Hence, we get $\mathscr{T}(P) \subset P$. By using standard arguments, we can easily show that $T_{1}$ and $T_{2}$ are completely continuous, and then $\mathscr{T}$ is a completely continuous operator.

For $c \in(0,1)$, we denote $A=\int_{0}^{1} J_{1}(s) d s, B=\int_{0}^{1} J_{2}(s) d s$, $C=\int_{0}^{1} J_{3}(s) d s, D=\int_{0}^{1} J_{4}(s) d s, \widetilde{A}=\int_{c}^{1} J_{1}(s) d s, \widetilde{B}=$ $\int_{c}^{1} J_{2}(s) d s, \widetilde{C}=\int_{c}^{1} J_{3}(s) d s$, and $\widetilde{D}=\int_{c}^{1} J_{4}(s) d s$, where $J_{i}, \quad i=$ $1, \ldots, 4$, are defined in Section 2 (Lemma 4).

For $f_{0}^{s}, g_{0}^{s}, f_{\infty}^{i}, g_{\infty}^{i} \in(0, \infty)$ and numbers $\alpha_{1}, \alpha_{2} \in[0,1]$, $\alpha_{3}, \alpha_{4} \in(0,1), a \in[0,1]$, and $b \in(0,1)$, we define the numbers

$$
\begin{aligned}
& L_{1}=\max \left\{\frac{a \alpha_{1}}{\gamma f_{\infty}^{i} \widetilde{A}}, \frac{(1-a) \alpha_{2}}{\gamma f_{\infty}^{i} \widetilde{D}}\right\}, \\
& L_{2}=\min \left\{\frac{b \alpha_{3}}{f_{0}^{s} A}, \frac{(1-b) \alpha_{4}}{f_{0}^{s} D}\right\}, \\
& L_{3}=\max \left\{\frac{a\left(1-\alpha_{1}\right)}{\gamma g_{\infty}^{i} \widetilde{B}}, \frac{(1-a)\left(1-\alpha_{2}\right)}{\gamma g_{\infty}^{i} \widetilde{C}}\right\}, \\
& L_{4}=\min \left\{\frac{b\left(1-\alpha_{3}\right)}{g_{0}^{s} B}, \frac{(1-b)\left(1-\alpha_{4}\right)}{g_{0}^{s} C}\right\}, \\
& L_{2}^{\prime}=\min \left\{\frac{b}{f_{0}^{s} A}, \frac{(1-b)}{f_{0}^{s} D}\right\}, \\
& L_{4}^{\prime}=\min \left\{\frac{b}{g_{0}^{s} B}, \frac{(1-b)}{g_{0}^{s} C}\right\},
\end{aligned}
$$

where $\gamma=\min \left\{c^{\alpha-1}, c^{\beta-1}\right\}$

Theorem 8. Assume that (H1) and (H2) hold, $c \in(0,1)$, $\alpha_{1}, \alpha_{2} \in[0,1], \alpha_{3}, \alpha_{4} \in(0,1)$, and $a \in[0,1]$ and $b \in(0,1)$.

(1) If $f_{0}^{s}, g_{0}^{s}, f_{\infty}^{i}, g_{\infty}^{i} \in(0, \infty), L_{1}<L_{2}$, and $L_{3}<L_{4}$, then, for each $\lambda \in\left(L_{1}, L_{2}\right)$ and $\mu \in\left(L_{3}, L_{4}\right)$, there exists a positive solution $(u(t), v(t)), t \in[0,1]$ for $(S)-(B C)$. 
(2) If $f_{0}^{s}=0, g_{0}^{s}, f_{\infty}^{i}, g_{\infty}^{i} \in(0, \infty)$ and $L_{3}<L_{4}^{\prime}$, then, for each $\lambda \in\left(L_{1}, \infty\right)$ and $\mu \in\left(L_{3}, L_{4}^{\prime}\right)$, there exists a positive solution $(u(t), v(t)), t \in[0,1]$ for $(S)-(B C)$.

(3) If $g_{0}^{s}=0, f_{0}^{s}, f_{\infty}^{i}, g_{\infty}^{i} \in(0, \infty)$, and $L_{1}<L_{2}^{\prime}$, then, for each $\lambda \in\left(L_{1}, L_{2}^{\prime}\right)$ and $\mu \in\left(L_{3}, \infty\right)$, there exists a positive solution $(u(t), v(t)), t \in[0,1]$ for $(S)-(B C)$.

(4) If $f_{0}^{s}=g_{0}^{s}=0, f_{\infty}^{i}, g_{\infty}^{i} \in(0, \infty)$, then, for each $\lambda \in\left(L_{1}, \infty\right)$ and $\mu \in\left(L_{3}, \infty\right)$, there exists a positive solution $(u(t), v(t)), t \in[0,1]$ for $(S)-(B C)$.

(5) If $\left\{f_{0}^{s}, g_{0}^{s}, f_{\infty}^{i} \in(0, \infty), g_{\infty}^{i}=\infty\right\}$ or $\left\{f_{0}^{s}, g_{0}^{s}, g_{\infty}^{i} \in\right.$ $\left.(0, \infty), f_{\infty}^{i}=\infty\right\}$ or $\left\{f_{0}^{s}, g_{0}^{s} \in(0, \infty), f_{\infty}^{i}=g_{\infty}^{i}=\infty\right\}$, then, for each $\lambda \in\left(0, L_{2}\right)$ and $\mu \in\left(0, L_{4}\right)$, there exists a positive solution $(u(t), v(t)), t \in[0,1]$ for $(S)-(B C)$.

(6) If $\left\{f_{0}^{s}=0, g_{0}^{s}, f_{\infty}^{i} \in(0, \infty), g_{\infty}^{i}=\infty\right\}$ or $\left\{f_{0}^{s}=0, f_{\infty}^{i}=\right.$ $\left.\infty, g_{0}^{s}, g_{\infty}^{i} \in(0, \infty)\right\}$ or $\left\{f_{0}^{s}=0, g_{0}^{s} \in(0, \infty), f_{\infty}^{i}=g_{\infty}^{i}=\infty\right\}$, then, for each $\lambda \in(0, \infty)$ and $\mu \in\left(0, L_{4}^{\prime}\right)$, there exists a positive solution $(u(t), v(t)), t \in[0,1]$ for $(S)-(B C)$.

(7) If $\left\{f_{0}^{s}, f_{\infty}^{i} \in(0, \infty), g_{0}^{s}=0, g_{\infty}^{i}=\infty\right\}$ or $\left\{f_{0}^{s}, g_{\infty}^{i} \in\right.$ $\left.(0, \infty), g_{0}^{s}=0, f_{\infty}^{i}=\infty\right\}$, or $\left\{f_{0}^{s} \in(0, \infty), g_{0}^{s}=0, f_{\infty}^{i}=\right.$ $\left.g_{\infty}^{i}=\infty\right\}$, then, for each $\lambda \in\left(0, L_{2}^{\prime}\right)$ and $\mu \in(0, \infty)$, there exists a positive solution $(u(t), v(t)), t \in[0,1]$ for $(S)-(B C)$.

(8) If $\left\{f_{0}^{s}=g_{0}^{s}=0, f_{\infty}^{i} \in(0, \infty), g_{\infty}^{i}=\infty\right\}$ or $\left\{f_{0}^{s}=g_{0}^{s}=\right.$ $\left.0, f_{\infty}^{i}=\infty, g_{\infty}^{i} \in(0, \infty)\right\}$ or $\left\{f_{0}^{s}=g_{0}^{s}=0, f_{\infty}^{i}=g_{\infty}^{i}=\infty\right\}$, then, for each $\lambda \in(0, \infty)$ and $\mu \in(0, \infty)$, there exists a positive solution $(u(t), v(t)), t \in[0,1]$ for $(S)-(B C)$.

Proof. We consider the above cone $P \subset Y$ and the operators $T_{1}, T_{2}$, and $\mathscr{T}$. Because the proofs of the above cases are similar, in what follows we will prove one of them, namely, Case (2). So, we suppose $f_{0}^{s}=0$ and $g_{0}^{s}, f_{\infty}^{i}, g_{\infty}^{i} \in(0, \infty)$. Let $\lambda \in\left(L_{1}, \infty\right)$ and $\mu \in\left(L_{3}, L_{4}^{\prime}\right)$. We choose $\widetilde{\alpha}_{3} \in\left(0,1-\mu g_{0}^{s} B / b\right)$ and $\widetilde{\alpha}_{4} \in\left(0,1-\mu g_{0}^{s} C /(1-b)\right)$. Let $\varepsilon>0$ be a positive number such that $\varepsilon<f_{\infty}^{\mathrm{i}}, \varepsilon<g_{\infty}^{i}$ and

$$
\begin{aligned}
& \frac{a \alpha_{1}}{\gamma\left(f_{\infty}^{i}-\varepsilon\right) \widetilde{A}} \leq \lambda, \\
& \frac{a\left(1-\alpha_{1}\right)}{\gamma\left(g_{\infty}^{i}-\varepsilon\right) \widetilde{B}} \leq \mu, \\
& \frac{(1-a) \alpha_{2}}{\gamma\left(f_{\infty}^{i}-\varepsilon\right) \widetilde{D}} \leq \lambda, \\
& \frac{(1-a)\left(1-\alpha_{2}\right)}{\gamma\left(g_{\infty}^{i}-\varepsilon\right) \widetilde{C}} \leq \mu, \\
& \frac{b \widetilde{\alpha}_{3}}{\varepsilon A} \geq \lambda, \\
& \frac{b\left(1-\widetilde{\alpha}_{3}\right)}{\left(g_{0}^{s}+\varepsilon\right) B} \geq \mu, \\
& \frac{(1-b) \widetilde{\alpha}_{4}}{\varepsilon D} \geq \lambda, \\
& \frac{(1-b)\left(1-\widetilde{\alpha}_{4}\right)}{\left(g_{0}^{s}+\varepsilon\right) C} \geq \mu \text {. }
\end{aligned}
$$

By using $(H 2)$ and the definitions of $f_{0}^{s}$ and $g_{0}^{s}$, we deduce that there exists $R_{1}>0$ such that $f(t, u, v) \leq \varepsilon(u+v)$ and $g(t, u, v) \leq\left(g_{0}^{s}+\varepsilon\right)(u+v)$ for all $t \in[0,1], u, v \in \mathbb{R}_{+}$with $0 \leq$ $u+v \leq R_{1}$. We define the set $\Omega_{1}=\left\{(u, v) \in Y,\|(u, v)\|_{Y}<R_{1}\right\}$. Now let $(u, v) \in P \cap \partial \Omega_{1}$, that is, $(u, v) \in P$ with $\|(u, v)\|_{Y}=R_{1}$ or equivalently $\|u\|+\|v\|=R_{1}$. Then $u(t)+v(t) \leq R_{1}$ for all $t \in[0,1]$, and by Lemma 4 , we obtain

$$
\begin{aligned}
T_{1}(u, v)(t)= & \lambda \int_{0}^{1} G_{1}(t, s) f(s, u(s), v(s)) d s \\
& +\mu \int_{0}^{1} G_{2}(t, s) g(s, u(s), v(s)) d s \\
\leq & \lambda \int_{0}^{1} J_{1}(s) f(s, u(s), v(s)) d s \\
& +\mu \int_{0}^{1} J_{2}(s) g(s, u(s), v(s)) d s \\
\leq & \lambda \int_{0}^{1} J_{1}(s) \varepsilon(u(s)+v(s)) d s \\
& +\mu \int_{0}^{1} J_{2}(s)\left(g_{0}^{s}+\varepsilon\right)(u(s)+v(s)) d s \\
\leq & \lambda \varepsilon \int_{0}^{1} J_{1}(s)(\|u\|+\|v\|) d s \\
& +\mu\left(g_{0}^{s}+\varepsilon\right) \int_{0}^{1} J_{2}(s)(\|u\|+\|v\|) d s \\
= & {\left[\lambda \varepsilon A+\mu\left(g_{0}^{s}+\varepsilon\right) B\right]\|(u, v)\|_{Y} } \\
\leq & {\left[b \widetilde{\alpha}_{3}+b\left(1-\widetilde{\alpha}_{3}\right)\right]\|(u, v)\|_{Y} } \\
= & b\|(u, v)\|_{Y}, \quad t \in[0,1] .
\end{aligned}
$$

Therefore, $\left\|T_{1}(u, v)\right\| \leq b\|(u, v)\|_{Y}$.

In a similar manner, we conclude

$$
\begin{aligned}
T_{2}(u, v)(t)= & \mu \int_{0}^{1} G_{3}(t, s) g(s, u(s), v(s)) d s \\
& +\lambda \int_{0}^{1} G_{4}(t, s) f(s, u(s), v(s)) d s \\
\leq & \mu \int_{0}^{1} J_{3}(s) g(s, u(s), v(s)) d s \\
& +\lambda \int_{0}^{1} J_{4}(s) f(s, u(s), v(s)) d s \\
\leq & \mu \int_{0}^{1} J_{3}(s)\left(g_{0}^{s}+\varepsilon\right)(u(s)+v(s)) d s \\
& +\lambda \int_{0}^{1} J_{4}(s) \varepsilon(u(s)+v(s)) d s \\
\leq & \mu\left(g_{0}^{s}+\varepsilon\right) \int_{0}^{1} J_{3}(s)(\|u\|+\|v\|) d s \\
& +\lambda \varepsilon \int_{0}^{1} J_{4}(s)(\|u\|+\|v\|) d s
\end{aligned}
$$




$$
\begin{aligned}
& =\left[\mu\left(g_{0}^{s}+\varepsilon\right) C+\lambda \varepsilon D\right]\|(u, v)\|_{Y} \\
& \leq\left[(1-b)\left(1-\widetilde{\alpha}_{4}\right)+(1-b) \widetilde{\alpha}_{4}\right]\|(u, v)\|_{Y} \\
& =(1-b)\|(u, v)\|_{Y}, \quad t \in[0,1] .
\end{aligned}
$$

Hence, $\left\|T_{2}(u, v)\right\| \leq(1-b)\|(u, v)\|_{Y}$.

Then, for $(u, v) \in P \cap \partial \Omega_{1}$, we deduce

$$
\begin{aligned}
\|\mathscr{T}(u, v)\|_{Y} & =\left\|T_{1}(u, v)\right\|+\left\|T_{2}(u, v)\right\| \\
& \leq b\|(u, v)\|_{Y}+(1-b)\|(u, v)\|_{Y} \\
& =\|(u, v)\|_{Y} .
\end{aligned}
$$

By the definitions of $f_{\infty}^{i}$ and $g_{\infty}^{i}$, there exists $\bar{R}_{2}>0$ such that $f(t, u, v) \geq\left(f_{\infty}^{i}-\varepsilon\right)(u+v)$ and $g(t, u, v) \geq\left(g_{\infty}^{i}-\varepsilon\right)(u+v)$ for all $u, v \geq 0$ with $u+v \geq \bar{R}_{2}$ and $t \in[c, 1]$. We consider $R_{2}=$ $\max \left\{2 R_{1}, \bar{R}_{2} / \gamma\right\}$ and we define $\Omega_{2}=\left\{(u, v) \in Y,\|(u, v)\|_{Y}<\right.$ $\left.R_{2}\right\}$. Then for $(u, v) \in P$ with $\|(u, v)\|_{Y}=R_{2}$, we obtain

$$
\begin{aligned}
u(t)+v(t) & \geq t^{\alpha-1}\|u\|+t^{\beta-1}\|v\| \\
& \geq c^{\alpha-1}\|u\|+c^{\beta-1}\|v\| \geq \gamma(\|u\|+\|v\|) \\
& =\gamma\|(u, v)\|_{Y}=\gamma R_{2} \geq \bar{R}_{2}, \quad \forall t \in[c, 1] .
\end{aligned}
$$

Then, by Lemma 4, we conclude

$$
\begin{aligned}
& T_{1}(u, v)(1) \\
& \geq \lambda \int_{0}^{1} J_{1}(s) f(s, u(s), v(s)) d s \\
& \quad+\mu \int_{0}^{1} J_{2}(s) g(s, u(s), v(s)) d s \\
& \geq \lambda \int_{c}^{1} J_{1}(s) f(s, u(s), v(s)) d s \\
& \quad+\mu \int_{c}^{1} J_{2}(s) g(s, u(s), v(s)) d s \\
& \geq \lambda \int_{c}^{1} J_{1}(s)\left(f_{\infty}^{i}-\varepsilon\right)(u(s)+v(s)) d s \\
& \quad+\mu \int_{c}^{1} J_{2}(s)\left(g_{\infty}^{i}-\varepsilon\right)(u(s)+v(s)) d s \\
& \geq \lambda \gamma\left(f_{\infty}^{i}-\varepsilon\right) \int_{c}^{1} J_{1}(s)\|(u, v)\|_{Y} d s \\
& \geq {\left[a \alpha_{1}+a\left(1-\alpha_{1}\right)\right]\|(u, v)\|_{Y}=a\|(u, v)\|_{Y} . } \\
&+\mu \gamma\left(g_{\infty}^{i}-\varepsilon\right) \int_{c}^{1} J_{2}(s)\|(u, v)\|_{Y} d s \\
&\left.\lambda \gamma\left(f_{\infty}^{i}-\varepsilon\right) \widetilde{A}+\mu \gamma\left(g_{\infty}^{i}-\varepsilon\right) \widetilde{B}\right]\|(u, v)\|_{Y} \\
&
\end{aligned}
$$

So, $\left\|T_{1}(u, v)\right\| \geq T_{1}(u, v)(1) \geq a\|(u, v)\|_{Y}$.
In a similar manner, we deduce

$$
\begin{aligned}
& T_{2}(u, v)(1) \\
& \geq \mu \int_{0}^{1} J_{3}(s) g(s, u(s), v(s)) d s \\
& +\lambda \int_{0}^{1} J_{4}(s) f(s, u(s), v(s)) d s \\
& \geq \mu \int_{c}^{1} J_{3}(s) g(s, u(s), v(s)) d s \\
& +\lambda \int_{c}^{1} J_{4}(s) f(s, u(s), v(s)) d s \\
& \geq \mu \int_{c}^{1} J_{3}(s)\left(g_{\infty}^{i}-\varepsilon\right)(u(s)+v(s)) d s \\
& +\lambda \int_{c}^{1} J_{4}(s)\left(f_{\infty}^{i}-\varepsilon\right)(u(s)+v(s)) d s \\
& \geq \mu \gamma\left(g_{\infty}^{i}-\varepsilon\right) \int_{c}^{1} J_{3}(s)\|(u, v)\|_{Y} d s \\
& +\lambda \gamma\left(f_{\infty}^{i}-\varepsilon\right) \int_{c}^{1} J_{4}(s)\|(u, v)\|_{Y} d s \\
& =\left[\mu \gamma\left(g_{\infty}^{i}-\varepsilon\right) \widetilde{C}+\lambda \gamma\left(f_{\infty}^{i}-\varepsilon\right) \widetilde{D}\right]\|(u, v)\|_{Y} \\
& \geq\left[(1-a)\left(1-\alpha_{2}\right)+(1-a) \alpha_{2}\right]\|(u, v)\|_{Y} \\
& =(1-a)\|(u, v)\|_{Y} \text {. }
\end{aligned}
$$

So, $\left\|T_{2}(u, v)\right\| \geq T_{2}(u, v)(1) \geq(1-a)\|(u, v)\|_{Y}$.

Hence, for $(u, v) \in P \cap \partial \Omega_{2}$, we obtain

$$
\begin{aligned}
\|\mathscr{T}(u, v)\|_{Y} & =\left\|T_{1}(u, v)\right\|+\left\|T_{2}(u, v)\right\| \\
& \geq a\|(u, v)\|_{Y}+(1-a)\|(u, v)\|_{Y} \\
& =\|(u, v)\|_{Y} .
\end{aligned}
$$

By using (17), (21), Lemma 7, and Theorem 6(i), we conclude that $\mathscr{T}$ has a fixed point $(u, v) \in P \cap\left(\bar{\Omega}_{2} \backslash \Omega_{1}\right)$ such that $R_{1} \leq\|u\|+\|v\| \leq R_{2}, u(t) \geq t^{\alpha-1}\|u\|$, and $v(t) \geq t^{\beta-1}\|v\|$ for all $t \in[0,1]$. If $\|u\|>0$ then $u(t)>0$ for all $t \in(0,1]$, and if $\|v\|>0$ then $v(t)>0$ for all $t \in(0,1]$.

In what follows, for $f_{0}^{i}, g_{0}^{i}, f_{\infty}^{s}, g_{\infty}^{s} \in(0, \infty)$ and numbers $\alpha_{1}, \alpha_{2} \in[0,1], \alpha_{3}, \alpha_{4} \in(0,1), a \in[0,1]$, and $b \in(0,1)$, we define the numbers

$$
\begin{aligned}
& \widetilde{L}_{1}=\max \left\{\frac{a \alpha_{1}}{\gamma f_{0}^{i} \widetilde{A}}, \frac{(1-a) \alpha_{2}}{\gamma f_{0}^{i} \widetilde{D}}\right\}, \\
& \widetilde{L}_{2}=\min \left\{\frac{b \alpha_{3}}{f_{\infty}^{s} A}, \frac{(1-b) \alpha_{4}}{f_{\infty}^{s} D}\right\}, \\
& \widetilde{L}_{3}=\max \left\{\frac{a\left(1-\alpha_{1}\right)}{\gamma g_{0}^{i} \widetilde{B}}, \frac{(1-a)\left(1-\alpha_{2}\right)}{\gamma g_{0}^{i} \widetilde{C}}\right\}, \\
& \widetilde{L}_{4}=\min \left\{\frac{b\left(1-\alpha_{3}\right)}{g_{\infty}^{s} B}, \frac{(1-b)\left(1-\alpha_{4}\right)}{g_{\infty}^{s} C}\right\},
\end{aligned}
$$




$$
\begin{aligned}
& \widetilde{L}_{2}^{\prime}=\min \left\{\frac{b}{f_{\infty}^{s} A}, \frac{1-b}{f_{\infty}^{s} D}\right\}, \\
& \widetilde{L}_{4}^{\prime}=\min \left\{\frac{b}{g_{\infty}^{s} B}, \frac{1-b}{g_{\infty}^{s} C}\right\} .
\end{aligned}
$$

Theorem 9. Assume that (H1) and (H2) hold, $c \in(0,1)$, $\alpha_{1}, \alpha_{2} \in[0,1], \alpha_{3}, \alpha_{4} \in(0,1), a \in[0,1]$, and $b \in(0,1)$.

(1) If $f_{0}^{i}, g_{0}^{i}, f_{\infty}^{s}, g_{\infty}^{s} \in(0, \infty)$ and $\widetilde{L}_{1}<\widetilde{L}_{2}$ and $\widetilde{L}_{3}<\widetilde{L}_{4}$, then, for each $\lambda \in\left(\widetilde{L}_{1}, \widetilde{L}_{2}\right)$ and $\mu \in\left(\widetilde{L}_{3}, \widetilde{L}_{4}\right)$, there exists a positive solution $(u(t), v(t)), t \in[0,1]$ for $(S)-(B C)$.

(2) If $f_{0}^{i}, g_{0}^{i}, f_{\infty}^{s} \in(0, \infty), g_{\infty}^{s}=0$, and $\widetilde{L}_{1}<\widetilde{L}_{2}^{\prime}$, then, for each $\lambda \in\left(\widetilde{L}_{1}, \widetilde{L}_{2}^{\prime}\right)$ and $\mu \in\left(\widetilde{L}_{3}, \infty\right)$, there exists a positive solution $(u(t), v(t)), t \in[0,1]$ for $(S)-(B C)$.

(3) If $f_{0}^{i}, g_{0}^{i}, g_{\infty}^{s} \in(0, \infty), f_{\infty}^{s}=0$, and $\widetilde{L}_{3}<\widetilde{L}_{4}^{\prime}$, then, for each $\lambda \in\left(\widetilde{L}_{1}, \infty\right)$ and $\mu \in\left(\widetilde{L}_{3}, \widetilde{L}_{4}^{\prime}\right)$, there exists a positive solution $(u(t), v(t)), t \in[0,1]$ for $(S)-(B C)$.

(4) If $f_{0}^{i}, g_{0}^{i} \in(0, \infty), f_{\infty}^{s}=g_{\infty}^{s}=0$, then for each $\lambda \in\left(\widetilde{L}_{1}, \infty\right)$ and $\mu \in\left(\widetilde{L}_{3}, \infty\right)$, there exists a positive solution $(u(t), v(t)), t \in[0,1]$ for $(S)-(B C)$.

(5) If $\left\{f_{0}^{i}=\infty, g_{0}^{i}, f_{\infty}^{s}, g_{\infty}^{s} \in(0, \infty)\right\}$ or $\left\{f_{0}^{i}, f_{\infty}^{s}, g_{\infty}^{s} \in\right.$ $\left.(0, \infty), g_{0}^{i}=\infty\right\}$ or $\left\{f_{0}^{i}=g_{0}^{i}=\infty, f_{\infty}^{s}, g_{\infty}^{s} \in(0, \infty)\right\}$, then, for each $\lambda \in\left(0, \widetilde{L}_{2}\right)$ and $\mu \in\left(0, \widetilde{L}_{4}\right)$, there exists a positive solution $(u(t), v(t)), t \in[0,1]$ for $(S)-(B C)$.

(6) If $\left\{f_{0}^{i}=\infty, g_{0}^{i}, f_{\infty}^{s} \in(0, \infty), g_{\infty}^{s}=0\right\}$ or $\left\{f_{0}^{i}, f_{\infty}^{s} \in\right.$ $\left.(0, \infty), g_{0}^{i}=\infty, g_{\infty}^{s}=0\right\}$ or $\left\{f_{0}^{i}=g_{0}^{i}=\infty, f_{\infty}^{s} \in\right.$ $\left.(0, \infty), g_{\infty}^{s}=0\right\}$, then, for each $\lambda \in\left(0, \widetilde{L}_{2}^{\prime}\right)$ and $\mu \in(0, \infty)$, there exists a positive solution $(u(t), v(t)), t \in[0,1]$ for $(S)$ $(B C)$.

(7) If $\left\{f_{0}^{i}=\infty, g_{0}^{i}, g_{\infty}^{s} \in(0, \infty), f_{\infty}^{s}=0\right\}$ or $\left\{f_{0}^{i}, g_{\infty}^{s} \in\right.$ $\left.(0, \infty), g_{0}^{i}=\infty, f_{\infty}^{s}=0\right\}$ or $\left\{f_{0}^{i}=g_{0}^{i}=\infty, f_{\infty}^{s}=0, g_{\infty}^{s} \in\right.$ $(0, \infty)\}$, then, for each $\lambda \in(0, \infty)$ and $\mu \in\left(0, \widetilde{L}_{4}^{\prime}\right)$, there exists a positive solution $(u(t), v(t)), t \in[0,1]$ for $(S)-(B C)$.

(8) If $\left\{f_{0}^{i}=\infty, g_{0}^{i} \in(0, \infty), f_{\infty}^{s}=g_{\infty}^{s}=0\right\}$ or $\left\{f_{0}^{i} \epsilon\right.$ $\left.(0, \infty), g_{0}^{i}=\infty, f_{\infty}^{s}=g_{\infty}^{s}=0\right\}$ or $\left\{f_{0}^{i}=g_{0}^{i}=\infty, f_{\infty}^{s}=g_{\infty}^{s}=\right.$ $0\}$, then, for each $\lambda \in(0, \infty)$ and $\mu \in(0, \infty)$, there exists a positive solution $(u(t), v(t)), t \in[0,1]$ for $(S)-(B C)$.

Proof. We consider again the above cone $P \subset Y$ and the operators $T_{1}, T_{2}$, and $\mathscr{T}$. Because the proofs of the above cases are similar, in what follows we will prove one of them, namely, the first case of (6). So, we suppose $f_{0}^{i}=\infty, g_{0}^{i}, f_{\infty}^{s} \in(0, \infty)$, and $g_{\infty}^{s}=0$. Let $\lambda \in\left(0, \widetilde{L}_{2}^{\prime}\right)$ and $\mu \in(0, \infty)$. We choose $\widetilde{\alpha}_{3} \in\left(\lambda f_{\infty}^{s} A / b, 1\right)$ and $\widetilde{\alpha}_{4} \in\left(\lambda f_{\infty}^{s} D /(1-b), 1\right)$, and let $\varepsilon>0$ be a positive number such that $\varepsilon<g_{0}^{i}, \varepsilon \leq \lambda \gamma \widetilde{A}$ and

$$
\begin{gathered}
\frac{b \widetilde{\alpha}_{3}}{\left(f_{\infty}^{s}+\varepsilon\right) A} \geq \lambda, \\
\frac{b\left(1-\widetilde{\alpha}_{3}\right)}{\varepsilon B} \geq \mu,
\end{gathered}
$$

$$
\begin{aligned}
\frac{(1-b) \tilde{\alpha}_{4}}{\left(f_{\infty}^{s}+\varepsilon\right) D} & \geq \lambda, \\
\frac{(1-b)\left(1-\widetilde{\alpha}_{4}\right)}{\varepsilon C} & \geq \mu .
\end{aligned}
$$

By using (H2) and the definitions of $f_{0}^{i}$ and $g_{0}^{i}$, we deduce that there exists $R_{3}>0$ such that $f(t, u, v) \geq(1 / \varepsilon)(u+v)$ and $g(t, u, v) \geq\left(g_{0}^{i}-\varepsilon\right)(u+v)$ for all $u, v \geq 0$ with $0 \leq u+v \leq R_{3}$ and $t \in[c, 1]$. We denote $\Omega_{3}=\left\{(u, v) \in Y,\|(u, v)\|_{Y}<R_{3}\right\}$. Let $(u, v) \in P$ with $\|(u, v)\|_{Y}=R_{3}$, that is, $\|u\|+\|v\|=R_{3}$. Because $u(t)+v(t) \leq\|u\|+\|v\|=R_{3}$ for all $t \in[0,1]$, then, by using Lemma 4 , we obtain

$$
\begin{aligned}
T_{1}(u, v)(1) \geq & \lambda \int_{0}^{1} J_{1}(s) f(s, u(s), v(s)) d s \\
& +\mu \int_{0}^{1} J_{2}(s) g(s, u(s), v(s)) d s \\
\geq & \lambda \int_{0}^{1} J_{1}(s) f(s, u(s), v(s)) d s \\
\geq & \lambda \int_{0}^{1} J_{1}(s) \frac{1}{\varepsilon}(u(s)+v(s)) d s \\
\geq & \frac{\lambda}{\varepsilon} \int_{c}^{1} J_{1}(s)\left(c^{\alpha-1}\|u\|+c^{\beta-1}\|v\|\right) d s \\
\geq & \frac{\lambda \gamma}{\varepsilon} \widetilde{A}\|(u, v)\|_{Y} \geq\|(u, v)\|_{Y} .
\end{aligned}
$$

Therefore, $\left\|T_{1}(u, v)\right\| \geq T_{1}(u, v)(1) \geq\|(u, v)\|_{Y}$.

Thus, for an arbitrary element $(u, v) \in P \cap \partial \Omega_{3}$, we deduce

$$
\begin{aligned}
\|\mathscr{T}(u, v)\|_{Y} & =\left\|T_{1}(u, v)\right\|+\left\|T_{2}(u, v)\right\| \geq\left\|T_{1}(u, v)\right\| \\
& \geq\|(u, v)\|_{Y} .
\end{aligned}
$$

Now, we define the functions $f^{*}, g^{*}:[0,1] \times$ $\mathbb{R}_{+} \rightarrow \mathbb{R}_{+}, f^{*}(t, x)=\max _{0 \leq u+v \leq x} f(t, u, v), g^{*}(t, x)=$ $\max _{0 \leq u+v \leq x} g(t, u, v), t \in[0,1], x \in \mathbb{R}_{+}$. Then $f(t, u, v) \leq$ $f^{*}(t, x), g(t, u, v) \leq g^{*}(t, x)$ for all $t \in[0,1], u \geq 0, v \geq 0$, and $u+v \leq x$. The functions $f^{*}(t, \cdot), g^{*}(t, \cdot)$ are nondecreasing for every $t \in[0,1]$, and they satisfy the conditions

$$
\begin{aligned}
& \limsup _{x \rightarrow \infty} \max _{t \in[0,1]} \frac{f^{*}(t, x)}{x} \leq f_{\infty}^{s}, \\
& \limsup _{x \rightarrow \infty} \max _{t \in[0,1]} \frac{g^{*}(t, x)}{x}=0 .
\end{aligned}
$$

Therefore, for $\varepsilon>0$, there exists $\bar{R}_{4}>0$ such that, for all $x \geq \bar{R}_{4}$ and $t \in[0,1]$, we have

$$
\begin{aligned}
& \frac{f^{*}(t, x)}{x} \leq \limsup _{x \rightarrow \infty} \max _{t \in[0,1]} \frac{f^{*}(t, x)}{x}+\varepsilon \leq f_{\infty}^{s}+\varepsilon, \\
& \frac{g^{*}(t, x)}{x} \leq \limsup _{x \rightarrow \infty} \max _{t \in[0,1]} \frac{g^{*}(t, x)}{x}+\varepsilon=\varepsilon,
\end{aligned}
$$

and so $f^{*}(t, x) \leq\left(f_{\infty}^{s}+\varepsilon\right) x$ and $g^{*}(t, x) \leq \varepsilon x$. 
We consider $R_{4}=\max \left\{2 R_{3}, \bar{R}_{4}\right\}$ and we denote $\Omega_{4}=$ $\left\{(u, v) \in Y,\|(u, v)\|_{Y}<R_{4}\right\}$. Let $(u, v) \in P \cap \partial \Omega_{4}$. By the definitions of $f^{*}$ and $g^{*}$, we obtain

$$
\begin{aligned}
& f(t, u(t), v(t)) \leq f^{*}\left(t,\|(u, v)\|_{Y}\right), \\
& g(t, u(t), v(t)) \leq g^{*}\left(t,\|(u, v)\|_{Y}\right),
\end{aligned}
$$

$\forall t \in[0,1]$.

Then, for all $t \in[0,1]$, we conclude

$$
\begin{aligned}
T_{1}(u, v)(t) \leq & \lambda \int_{0}^{1} J_{1}(s) f(s, u(s), v(s)) d s \\
& +\mu \int_{0}^{1} J_{2}(s) g(s, u(s), v(s)) d s \\
\leq & \lambda \int_{0}^{1} J_{1}(s) f^{*}\left(s,\|(u, v)\|_{Y}\right) d s \\
& +\mu \int_{0}^{1} J_{2}(s) g^{*}\left(s,\|(u, v)\|_{Y}\right) d s \\
\leq & \lambda\left(f_{\infty}^{s}+\varepsilon\right) \int_{0}^{1} J_{1}(s)\|(u, v)\|_{Y} d s \\
& +\mu \varepsilon \int_{0}^{1} J_{2}(s)\|(u, v)\|_{Y} d s \\
= & {\left[\lambda\left(f_{\infty}^{s}+\varepsilon\right) A+\mu \varepsilon B\right]\|(u, v)\|_{Y} } \\
\leq & {\left[b \widetilde{\alpha}_{3}+b\left(1-\widetilde{\alpha}_{3}\right)\right]\|(u, v)\|_{Y} } \\
= & b\|(u, v)\|_{Y} .
\end{aligned}
$$

Therefore, $\left\|T_{1}(u, v)\right\| \leq b\|(u, v)\|_{Y}$.

In a similar manner, we deduce

$$
\begin{aligned}
T_{2}(u, v)(t) \leq & \mu \int_{0}^{1} J_{3}(s) g(s, u(s), v(s)) d s \\
& +\lambda \int_{0}^{1} J_{4}(s) f(s, u(s), v(s)) d s \\
\leq & \mu \int_{0}^{1} J_{3}(s) g^{*}\left(s,\|(u, v)\|_{Y}\right) d s \\
& +\lambda \int_{0}^{1} J_{4}(s) f^{*}\left(s,\|(u, v)\|_{Y}\right) d s \\
\leq & \mu \varepsilon \int_{0}^{1} J_{3}(s)\|(u, v)\|_{Y} d s \\
& +\lambda\left(f_{\infty}^{s}+\varepsilon\right) \int_{0}^{1} J_{4}(s)\|(u, v)\|_{Y} d s \\
= & {\left[\mu \varepsilon C+\lambda\left(f_{\infty}^{s}+\varepsilon\right) D\right]\|(u, v)\|_{Y} }
\end{aligned}
$$

$$
\begin{aligned}
& \leq\left[(1-b)\left(1-\widetilde{\alpha}_{4}\right)+(1-b) \widetilde{\alpha}_{4}\right]\|(u, v)\|_{Y} \\
& =(1-b)\|(u, v)\|_{Y} .
\end{aligned}
$$

So, $\left\|T_{2}(u, v)\right\| \leq(1-b)\|(u, v)\|_{Y}$.

Then, for $(u, v) \in P \cap \partial \Omega_{4}$, it follows that

$$
\begin{aligned}
\|\mathscr{T}(u, v)\|_{Y} & =\left\|T_{1}(u, v)\right\|+\left\|T_{2}(u, v)\right\| \\
& \leq b\|(u, v)\|_{Y}+(1-b)\|(u, v)\|_{Y} \\
& =\|(u, v)\|_{Y} .
\end{aligned}
$$

By using (25), (31), Lemma 7, and Theorem 6(ii), we conclude that $\mathscr{T}$ has a fixed point $(u, v) \in P \cap\left(\bar{\Omega}_{4} \backslash \Omega_{3}\right)$ such that $R_{3} \leq\|(u, v)\|_{Y} \leq R_{4}$.

\section{Nonexistence Results for the Positive Solutions}

We present in this section intervals for $\lambda$ and $\mu$ for which there exists no positive solution of problem $(S)-(B C)$ that can be viewed as fixed point of operator $\mathscr{T}$.

Theorem 10. Assume that (H1) and (H2) hold, and $c \in(0,1)$. If $f_{0}^{s}, f_{\infty}^{s}, g_{0}^{s}, g_{\infty}^{s}<\infty$, then there exist positive constants $\lambda_{0}, \mu_{0}$ such that, for every $\lambda \in\left(0, \lambda_{0}\right)$ and $\mu \in\left(0, \mu_{0}\right)$, the boundary value problem $(S)-(B C)$ has no positive solution.

Proof. In a similar manner as in the proof of Theorem 3.1 from [11], we can show that $\lambda_{0}=\min \left\{1 /\left(4 M_{1} A\right), 1 /\left(4 M_{1} D\right)\right\}$ and $\mu_{0}=\min \left\{1 /\left(4 M_{2} B\right), 1 /\left(4 M_{2} C\right)\right\}$, where $A=\int_{0}^{1} J_{1}(s) d s$, $B=\int_{0}^{1} J_{2}(s) d s, C=\int_{0}^{1} J_{3}(s) d s$, and $D=\int_{0}^{1} J_{4}(s) d s$, satisfy the conditions of our theorem.

Theorem 11. Assume that (H1) and (H2) hold, and $c \in(0,1)$. If $f_{0}^{i}, f_{\infty}^{i}>0$ and $f(t, u, v)>0$ for all $t \in[c, 1], u \geq 0, v \geq 0$, and $u+v>0$, then there exists a positive constant $\widetilde{\lambda}_{0}$ such that, for every $\lambda>\tilde{\lambda}_{0}$ and $\mu>0$, the boundary value problem $(S)-(B C)$ has no positive solution.

Proof. From the assumptions of the theorem, we deduce that there exists $m_{1}>0$ such that $f(t, u, v) \geq m_{1}(u+$ $v)$ for all $t \in[c, 1]$ and $u, v \geq 0$. We define $\tilde{\lambda}_{0}=$ $\min \left\{1 /\left(\gamma m_{1} \widetilde{A}\right), 1 /\left(\gamma m_{1} \widetilde{D}\right)\right\}$, where $\widetilde{A}=\int_{c}^{1} J_{1}(s) d s$ and $\widetilde{D}=$ $\int_{c}^{1} J_{4}(s) d s$. We will show that, for every $\lambda>\tilde{\lambda}_{0}$ and $\mu>0$, problem $(S)-(B C)$ has no positive solution.

Let $\lambda>\widetilde{\lambda}_{0}$ and $\mu>0$. We suppose that $(S)-(B C)$ has a positive solution $(u(t), v(t)), t \in[0,1]$.

If $\widetilde{A} \geq \widetilde{D}$, then $\widetilde{\lambda}_{0}=1 /\left(\gamma m_{1} \widetilde{A}\right)$, and therefore, we obtain

$$
\begin{aligned}
u(1)= & \left(T_{1}(u, v)\right)(1) \\
= & \lambda \int_{0}^{1} G_{1}(1, s) f(s, u(s), v(s)) d s \\
& +\mu \int_{0}^{1} G_{2}(1, s) g(s, u(s), v(s)) d s
\end{aligned}
$$




$$
\begin{aligned}
& \geq \lambda \int_{0}^{1} G_{1}(1, s) f(s, u(s), v(s)) d s \\
& \geq \lambda \int_{c}^{1} G_{1}(1, s) f(s, u(s), v(s)) d s \\
& \geq \lambda m_{1} \int_{c}^{1} G_{1}(1, s)(u(s)+v(s)) d s \\
& \geq \lambda m_{1} \int_{c}^{1} J_{1}(s)(u(s)+v(s)) d s \\
& \geq \lambda m_{1} \gamma \int_{c}^{1} J_{1}(s)(\|u\|+\|v\|) d s \\
& =\lambda m_{1} \gamma \widetilde{A}\|(u, v)\|_{Y} .
\end{aligned}
$$

Then we conclude

$$
\begin{aligned}
\|u\| & \geq u(1) \geq \lambda m_{1} \gamma \widetilde{A}\|(u, v)\|_{Y}>\widetilde{\lambda}_{0} m_{1} \gamma \widetilde{A}\|(u, v)\|_{Y} \\
& =\|(u, v)\|_{Y},
\end{aligned}
$$

and so, $\|(u, v)\|_{Y}=\|u\|+\|v\| \geq\|u\|>\|(u, v)\|_{Y}$, which is a contradiction.

If $\widetilde{A}<\widetilde{D}$, then $\widetilde{\lambda}_{0}=1 /\left(\gamma m_{1} \widetilde{D}\right)$, and therefore, we deduce

$$
\begin{aligned}
v(1)= & \left(T_{2}(u, v)\right)(1) \\
= & \mu \int_{0}^{1} G_{3}(1, s) g(s, u(s), v(s)) d s \\
& +\lambda \int_{0}^{1} G_{4}(1, s) f(s, u(s), v(s)) d s \\
\geq & \lambda \int_{0}^{1} G_{4}(1, s) f(s, u(s), v(s)) d s \\
\geq & \lambda \int_{c}^{1} G_{4}(1, s) f(s, u(s), v(s)) d s \\
\geq & \lambda m_{1} \int_{c}^{1} G_{4}(1, s)(u(s)+v(s)) d s \\
\geq & \lambda m_{1} \int_{c}^{1} J_{4}(s)(u(s)+v(s)) d s \\
\geq & \lambda m_{1} \gamma \int_{c}^{1} J_{4}(s)(\|u\|+\|v\|) d s \\
= & \lambda m_{1} \gamma \widetilde{D}\|(u, v)\|_{Y} .
\end{aligned}
$$

Then we conclude

$$
\begin{aligned}
\|v\| & \geq v(1) \geq \lambda m_{1} \gamma \widetilde{D}\|(u, v)\|_{Y}>\widetilde{\lambda}_{0} m_{1} \gamma \widetilde{D}\|(u, v)\|_{Y} \\
& =\|(u, v)\|_{Y},
\end{aligned}
$$

and so, $\|(u, v)\|_{Y}=\|u\|+\|v\| \geq\|v\|>\|(u, v)\|_{Y}$, which is a contradiction.

Therefore, the boundary value problem $(S)-(B C)$ has no positive solution.
Theorem 12. Assume that $(H 1)$ and $(H 2)$ hold, and $c \in(0,1)$. If $g_{0}^{i}, g_{\infty}^{i}>0$ and $g(t, u, v)>0$ for all $t \in[c, 1], u \geq 0, v \geq 0$, and $u+v>0$, then there exists a positive constant $\tilde{\mu}_{0}$ such that, for every $\mu>\widetilde{\mu}_{0}$ and $\lambda>0$, the boundary value problem $(S)-(B C)$ has no positive solution.

Proof. From the assumptions of the theorem, we deduce that there exists $m_{2}>0$ such that $g(t, u, v) \geq m_{2}(u+v)$ for all $t \in$ $[c, 1]$ and $u, v \geq 0$. We define $\widetilde{\mu}_{0}=\min \left\{1 /\left(\gamma m_{2} \widetilde{B}\right), 1 /\left(\gamma m_{2} \widetilde{C}\right)\right\}$, where $\widetilde{B}=\int_{c}^{1} J_{2}(s) d s$ and $\widetilde{C}=\int_{c}^{1} J_{3}(s) d s$. Using a similar approach as that used in the proof of Theorem 11, we can show that, for every $\mu>\widetilde{\mu}_{0}$ and $\lambda>0$, problem $(S)-(B C)$ has no positive solution.

Theorem 13. Assume that (H1) and (H2) hold, and $c \in(0,1)$. If $f_{0}^{i}, f_{\infty}^{i}, g_{0}^{i}, g_{\infty}^{i}>0$ and $f(t, u, v)>0, g(t, u, v)>0$ for all $t \in[c, 1], u \geq 0, v \geq 0$, and $u+v>0$, then there exist positive constants $\hat{\lambda}_{0}$ and $\hat{\mu}_{0}$ such that, for every $\lambda>\widehat{\lambda}_{0}$ and $\mu>\widehat{\mu}_{0}$, the boundary value problem $(S)-(B C)$ has no positive solution.

Proof. From the assumptions of the theorem, we deduce that there exist $m_{1}, m_{2}>0$ such that $f(t, u, v) \geq m_{1}(u+v)$ and $g(t, u, v) \geq m_{2}(u+v)$, for all $t \in[c, 1]$ and $u, v \geq 0$.

We define $\widehat{\lambda}_{0}=1 /\left(2 \gamma m_{1} \widetilde{A}\right)$ and $\widehat{\mu}_{0}=1 /\left(2 \gamma m_{2} \widetilde{C}\right)$, where $\widetilde{A}=\int_{c}^{1} J_{1}(s) d s$ and $\widetilde{C}=\int_{c}^{1} J_{3}(s) d s$. Then, for every $\lambda>\widehat{\lambda}_{0}$ and $\mu>\widehat{\mu}_{0}$, problem $(S)-(B C)$ has no positive solution. Indeed, let $\lambda>\widehat{\lambda}_{0}$ and $\mu>\widehat{\mu}_{0}$. We suppose that $(S)-(B C)$ has a positive solution $(u(t), v(t)), t \in[0,1]$. In a similar manner as that used in the proofs of Theorems 11 and 12, we obtain

$$
\begin{gathered}
\|u\| \geq u(1) \geq \lambda m_{1} \gamma \widetilde{A}\|(u, v)\|_{Y}, \\
\|v\| \geq v(1) \geq \mu m_{2} \gamma \widetilde{C}\|(u, v)\|_{Y},
\end{gathered}
$$

and so

$$
\begin{aligned}
\|(u, v)\|_{Y} & =\|u\|+\|v\| \\
& \geq \lambda m_{1} \gamma \widetilde{A}\|(u, v)\|_{Y}+\mu m_{2} \gamma \widetilde{C}\|(u, v)\|_{Y} \\
& >\widehat{\lambda}_{0} m_{1} \gamma \widetilde{A}\|(u, v)\|_{Y}+\widehat{\mu}_{0} m_{2} \gamma \widetilde{C}\|(u, v)\|_{Y} \\
& =\frac{1}{2}\|(u, v)\|_{Y}+\frac{1}{2}\|(u, v)\|_{Y}=\|(u, v)\|_{Y}
\end{aligned}
$$

which is a contradiction. Therefore, the boundary value problem $(S)-(B C)$ has no positive solution.

We can also define $\hat{\lambda}_{0}^{\prime}=1 /\left(2 \gamma m_{1} \widetilde{D}\right)$ and $\widehat{\mu}_{0}^{\prime}=1 /\left(2 \gamma m_{2} \widetilde{B}\right)$, where $\widetilde{B}=\int_{c}^{1} J_{2}(s) d s$ and $\widetilde{D}=\int_{c}^{1} J_{4}(s) d s$. Then, for every $\lambda>\hat{\lambda}_{0}^{\prime}$ and $\mu>\widehat{\mu}_{0}^{\prime}$, problem $(S)-(B C)$ has no positive solution. Indeed, let $\lambda>\widehat{\lambda}_{0}^{\prime}$ and $\mu>\widehat{\mu}_{0}^{\prime}$. We suppose that $(S)-(B C)$ has a positive solution $(u(t), v(t)), t \in[0,1]$. In a similar manner as that used in the proofs of Theorems 11 and 12, we obtain

$$
\begin{gathered}
\|v\| \geq v(1) \geq \lambda m_{1} \gamma \widetilde{D}\|(u, v)\|_{Y}, \\
\|u\| \geq u(1) \geq \mu m_{2} \gamma \widetilde{B}\|(u, v)\|_{Y},
\end{gathered}
$$


and so

$$
\begin{aligned}
\|(u, v)\|_{Y} & =\|u\|+\|v\| \\
& \geq \mu m_{2} \gamma \widetilde{B}\|(u, v)\|_{Y}+\lambda m_{1} \gamma \widetilde{D}\|(u, v)\|_{Y} \\
& >\widehat{\mu}_{0}^{\prime} m_{2} \gamma \widetilde{B}\|(u, v)\|_{Y}+\widehat{\lambda}_{0}^{\prime} m_{1} \gamma \widetilde{D}\|(u, v)\|_{Y} \\
& =\frac{1}{2}\|(u, v)\|_{Y}+\frac{1}{2}\|(u, v)\|_{Y}=\|(u, v)\|_{Y},
\end{aligned}
$$

which is a contradiction. Therefore, the boundary value problem $(S)-(B C)$ has no positive solution.

\section{Examples}

Let $\alpha=5 / 2(n=3), \beta=7 / 3(m=3), H(t)=t^{3}$, $K(t)=\{0, t \in[0,1 / 3) ; 1, t \in[1 / 3,2 / 3) ; 2, t \in[2 / 3,1]\}$, for all $t \in[0,1]$. Then $\int_{0}^{1} u(s) d K(s)=u(1 / 3)+u(2 / 3)$ and $\int_{0}^{1} v(s) d H(s)=3 \int_{0}^{1} s^{2} v(s) d s$.

We consider the system of fractional differential equations

$$
\begin{aligned}
& D_{0+}^{5 / 2} u(t)+\lambda f(t, u(t), v(t))=0, \quad t \in(0,1), \\
& D_{0+}^{7 / 3} v(t)+\mu g(t, u(t), v(t))=0, \quad t \in(0,1),
\end{aligned}
$$

with the boundary conditions

$$
\begin{gathered}
u(0)=u^{\prime}(0)=0, \\
u^{\prime}(1)=3 \int_{0}^{1} s^{2} v(s) d s,
\end{gathered}
$$

$$
\begin{aligned}
v(0) & =v^{\prime}(0)=0, \\
v^{\prime}(1) & =u\left(\frac{1}{3}\right)+u\left(\frac{2}{3}\right) .
\end{aligned}
$$

Then we obtain $\Delta=(\alpha-1)(\beta-1)-$ $\left(\int_{0}^{1} s^{\alpha-1} d K(s)\right)\left(\int_{0}^{1} s^{\beta-1} d H(s)\right)=(26 \sqrt{3}-6 \sqrt{2}-3) / 13 \sqrt{3}>0$. The functions $H$ and $K$ are nondecreasing, and so assumption $(H 1)$ is satisfied. Besides, we deduce

$$
\begin{aligned}
& g_{1}(t, s)=\frac{1}{\Gamma(5 / 2)} \\
& \qquad \begin{cases}t^{3 / 2}(1-s)^{1 / 2}-(t-s)^{3 / 2}, & 0 \leq s \leq t \leq 1, \\
t^{3 / 2}(1-s)^{1 / 2}, & 0 \leq t \leq s \leq 1,\end{cases} \\
& g_{2}(t, s)=\frac{1}{\Gamma(7 / 3)} \\
& \qquad \begin{cases}t^{4 / 3}(1-s)^{1 / 3}-(t-s)^{4 / 3}, & 0 \leq s \leq t \leq 1, \\
t^{4 / 3}(1-s)^{1 / 3}, & 0 \leq t \leq s \leq 1,\end{cases}
\end{aligned}
$$

$h_{1}(s)=\frac{s(1-s)^{1 / 2}}{\Gamma(5 / 2)}$,

$h_{2}(s)=\frac{s(1-s)^{1 / 3}}{\Gamma(7 / 3)}$.

We also obtain

$$
\begin{aligned}
& J_{1}(s)= \begin{cases}\frac{4}{3 \sqrt{\pi}} s(1-s)^{1 / 2}+\frac{4}{(26 \sqrt{3}-6 \sqrt{2}-3) \sqrt{\pi}}\left[(1+2 \sqrt{2})(1-s)^{1 / 2}-(1-3 s)^{3 / 2}-(2-3 s)^{3 / 2}\right], & 0 \leq s<\frac{1}{3}, \\
\frac{4}{3 \sqrt{\pi}} s(1-s)^{1 / 2}+\frac{4}{(26 \sqrt{3}-6 \sqrt{2}-3) \sqrt{\pi}}\left[(1+2 \sqrt{2})(1-s)^{1 / 2}-(2-3 s)^{3 / 2}\right], & \frac{1}{3} \leq s<\frac{2}{3}, \\
\frac{4}{3 \sqrt{\pi}} s(1-s)^{1 / 2}+\frac{4}{(26 \sqrt{3}-6 \sqrt{2}-3) \sqrt{\pi}}(1+2 \sqrt{2})(1-s)^{1 / 2}, & \frac{2}{3} \leq s \leq 1,\end{cases} \\
& J_{2}(s)=\frac{52 \sqrt{3}}{(26 \sqrt{3}-6 \sqrt{2}-3) \Gamma(7 / 3)}\left[\frac{3}{13}(1-s)^{1 / 3}-\frac{3}{13}(1-s)^{13 / 3}-\frac{3}{5} s(1-s)^{10 / 3}-\frac{3}{7} s^{2}(1-s)^{7 / 3}\right], \\
& J_{3}(s)=\frac{s(1-s)^{1 / 3}}{\Gamma(7 / 3)}+\frac{13(1+2 \sqrt{2})}{(26 \sqrt{3}-6 \sqrt{2}-3) \Gamma(7 / 3)}\left[\frac{3}{13}(1-s)^{1 / 3}-\frac{3}{13}(1-s)^{13 / 3}-\frac{3}{5} s(1-s)^{10 / 3}-\frac{3}{7} s^{2}(1-s)^{7 / 3}\right], \\
& J_{4}(s)=\frac{26}{3(26 \sqrt{3}-6 \sqrt{2}-3) \sqrt{\pi} \begin{cases}(1+2 \sqrt{2})(1-s)^{1 / 2}-(1-3 s)^{3 / 2}-(2-3 s)^{3 / 2}, & 0 \leq s<\frac{1}{3}, \\
(1+2 \sqrt{2})(1-s)^{1 / 2}-(2-3 s)^{3 / 2}, & \frac{1}{3} \leq s<\frac{2}{3},\end{cases} } \begin{array}{ll}
\frac{2}{3} \leq s \leq 1 .
\end{array}
\end{aligned}
$$


For $c=1 / 2$, we deduce $\gamma=1 /(2 \sqrt{2})$. After some computations, we conclude $A=\int_{0}^{1} J_{1}(s) d s \approx 0.31258448$, $\widetilde{A}=\int_{1 / 2}^{1} J_{1}(s) d s \approx 0.18323168, B=\int_{0}^{1} J_{2}(s) d s \approx 0.20906868$, $\widetilde{B}=\int_{1 / 2}^{1} J_{2}(s) d s \approx 0.13212807, C=\int_{0}^{1} J_{3}(s) d s \approx 0.38549139$, $\widetilde{C}=\int_{1 / 2}^{1} J_{3}(s) d s \approx 0.25157025, D=\int_{0}^{1} J_{4}(s) d s \approx 0.24263144$, and $\widetilde{D}=\int_{1 / 2}^{1} J_{4}(s) d s \approx 0.12808534$.

Example 1. We consider the functions

$$
\begin{aligned}
& f(t, u, v)=\frac{\sqrt{t}\left[p_{1}(u+v)+1\right](u+v)\left(q_{1}+\sin v\right)}{u+v+1}, \\
& g(t, u, v)=\frac{\sqrt[3]{t}\left[p_{2}(u+v)+1\right](u+v)\left(q_{2}+\cos u\right)}{u+v+1},
\end{aligned}
$$

for $t \in[0,1], u, v \geq 0$, where $p_{1}, p_{2}>0$ and $q_{1}, q_{2}>1$.

We have $f_{0}^{s}=q_{1}, g_{0}^{s}=q_{2}+1, f_{\infty}^{i}=(1 / \sqrt{2}) p_{1}\left(q_{1}-1\right)$, and $g_{\infty}^{i}=(1 / \sqrt[3]{2}) p_{2}\left(q_{2}-1\right)$. We take $a=b=\alpha_{1}=\alpha_{2}=$ $\alpha_{3}=\alpha_{4}=1 / 2$; then we obtain $L_{1}=1 /\left(p_{1}\left(q_{1}-1\right) \widetilde{D}\right), L_{2}=$ $1 /\left(4 q_{1} A\right), L_{3}=1 /\left(2^{1 / 6} p_{2}\left(q_{2}-1\right) \widetilde{B}\right)$, and $L_{4}=1 /\left(4\left(q_{2}+1\right) C\right)$. The conditions $L_{1}<L_{2}$ and $L_{3}<L_{4}$ become $p_{1}\left(q_{1}-1\right) / q_{1}>$ $4 A / \widetilde{D}$ and $p_{2}\left(q_{2}-1\right) /\left(q_{2}+1\right)>2{ }^{11 / 6} C / \widetilde{B}$. If $p_{1}\left(q_{1}-1\right) / q_{1} \geq 10$ and $p_{2}\left(q_{2}-1\right) /\left(q_{2}+1\right) \geq 11$, then the above conditions are satisfied. Therefore, by Theorem $8(1)$, for each $\lambda \in\left(L_{1}, L_{2}\right)$ and $\mu \in\left(L_{3}, L_{4}\right)$, there exists a positive solution $(u(t), v(t))$, $t \in[0,1]$ for problem $\left(S_{0}\right)-\left(B C_{0}\right)$. For example, if $q_{1}=2, q_{2}=$ 3 , $p_{1}=20$, and $p_{2}=22$, then we obtain $L_{1} \approx 0.39036474$, $L_{2} \approx 0.39989189, L_{3} \approx 0.15324297$, and $L_{4} \approx 0.16213072$.

Besides, because $f_{0}^{s}=q_{1}, g_{0}^{s}=q_{2}+1, f_{\infty}^{s}=$ $p_{1}\left(q_{1}+1\right)$, and $g_{\infty}^{s}=p_{2}\left(q_{2}+1\right)$, we can apply Theorem 10 . So, we conclude that there exist $\lambda_{0}, \mu_{0}>0$ such that, for every $\lambda \in\left(0, \lambda_{0}\right)$ and $\mu \in\left(0, \mu_{0}\right)$, the boundary value problem $\left(S_{0}\right)-\left(B C_{0}\right)$ has no positive solution. By Theorem 10, the positive constants $\lambda_{0}$ and $\mu_{0}$ are given by $\lambda_{0}=\min \left\{1 /\left(4 M_{1} A\right), 1 /\left(4 M_{1} D\right)\right\}=1 /\left(4 M_{1} A\right)$ and $\mu_{0}=$ $\min \left\{1 /\left(4 M_{2} B\right), 1 /\left(4 M_{2} C\right)\right\}=1 /\left(4 M_{2} C\right)$. For example, if $p_{1}=20, p_{2}=22, q_{1}=2$, and $q_{2}=3$, then we obtain $M_{1}=60$, $M_{2}=88, \lambda_{0} \approx 13.33 \cdot 10^{-3}$, and $\mu_{0} \approx 7.37 \cdot 10^{-3}$.

Example 2. We consider the functions

$$
\begin{aligned}
& f(t, u, v)=(t+1)^{\tilde{a}}\left(u^{2}+v^{2}\right), \\
& g(t, u, v)=t^{\tilde{b}}\left(e^{u+v}-1\right),
\end{aligned}
$$

$$
t \in[0,1], u, v \geq 0
$$

where $\tilde{a}, \tilde{b}>0$.

We have $f_{0}^{s}=0, g_{0}^{s}=1$, and $f_{\infty}^{i}=g_{\infty}^{i}=\infty$. For $b=1 / 2$, we obtain $L_{4}^{\prime}=1 /(2 C) \approx 1.2970458$. Then, by Theorem 8 , (6) we conclude that, for each $\lambda \in(0, \infty)$ and $\mu \in\left(0, L_{4}^{\prime}\right)$, there exists a positive solution $(u(t), v(t)), t \in[0,1]$ for problem $\left(S_{0}\right)-\left(B C_{0}\right)$.

Because $g_{0}^{i}=2^{-\widetilde{b}}$ and $g_{\infty}^{i}=\infty$, we can apply Theorem 12 . Then there exists $\widetilde{\mu}_{0}$ such that, for every $\mu>\widetilde{\mu}_{0}$ and $\lambda>0$, problem $\left(S_{0}\right)-\left(B C_{0}\right)$ has no positive solution. For example, if $\widetilde{b}=1$, then we deduce $m_{2}=1 / 2$ and $\widetilde{\mu}_{0}=1 /\left(\gamma m_{2} \widetilde{C}\right) \approx$ 22.486 .

\section{Competing Interests}

The authors declare that there is no conflict of interests regarding the publication of this paper and regarding the funding that they have received.

\section{Acknowledgments}

The work of Rodica Luca and Alexandru Tudorache was supported by the CNCS Grant PN-II-ID-PCE-2011-3-0557, Romania.

\section{References}

[1] S. Das, Functional Fractional Calculus for System Identification and Controls, Springer, New York, NY, USA, 2008.

[2] A. A. Kilbas, H. M. Srivastava, and J. J. Trujillo, Theory and Applications of Fractional Differential Equations, vol. 204 of North-Holland Mathematics Studies, Elsevier Science, Amsterdam, The Netherlands, 2006.

[3] I. Podlubny, Fractional Differential Equations, Academic Press, San Diego, Calif, USA, 1999.

[4] J. Sabatier, O. P. Agrawal, and J. A. T. Machado, Eds., Advances in Fractional Calculus: The-oretical Developments and Applications in Physics and Engineering, Springer, Dordrecht, The Netherlands, 2007.

[5] S. G. Samko, A. A. Kilbas, and O. I. Marichev, Fractional Integrals and Derivatives, Theory and Applications, Gordon and Breach, Yverdon, Switzerland, 1993.

[6] J. R. Cannon, "The solution of the heat equation subject to the specification of energy," Quarterly of Applied Mathematics, vol. 21, no. 3, pp. 155-160, 1963.

[7] R. Y. Chegi, "Numerical solution of the heat conduction problem with an integral condition," Litovskii Matematiceskii Sbornik, vol. 24, pp. 209-215, 1984.

[8] N. I. Ionkin, "Solution of a boundary-value problem in heat conduction with a nonclassical boundary condition," Differential Equations, vol. 13, no. 2, pp. 204-211, 1977.

[9] A. A. Samarskii, "Some problems of the theory of differential equations," Differentsial'nye Uravneniya, vol. 16, no. 11, pp. 19251935,1980 .

[10] J. Henderson and R. Luca, "Positive solutions for a system of fractional differential equations with coupled integral boundary conditions," Applied Mathematics and Computation, vol. 249, pp. 182-197, 2014.

[11] J. Henderson and R. Luca, "Nonexistence of positive solutions for a system of coupled fractional boundary value problems," Boundary Value Problems, vol. 2015, article 138, pp. 1-12, 2015.

[12] J. Henderson, R. Luca, and A. Tudorache, "On a system of fractional differential equations with coupled integral boundary conditions," Fractional Calculus and Applied Analysis, vol. 18, no. 2, pp. 361-386, 2015.

[13] J. Henderson and R. Luca, "Existence of positive solutions for a system of semipositone fractional boundary value problems," Electronic Journal of Qualitative Theory of Differential Equations, no. 22, pp. 1-28, 2016. 
[14] C. Yuan, D. Jiang, D. O'Regan, and R. P. Agarwal, "Multiple positive solutions to systems of nonlinear semipositone fractional differential equations with coupled boundary conditions," Electronic Journal of Qualitative Theory of Differential Equations, vol. 13, no. 13, pp. 1-17, 2012.

[15] J. Jiang, L. Liu, and Y. Wu, "Symmetric positive solutions to singular system with multi-point coupled boundary conditions," Applied Mathematics and Computation, vol. 220, pp. 536-548, 2013.

[16] J. Jiang, L. Liu, and Y. Wu, "Positive solutions to singular fractional differential system with coupled boundary conditions," Communications in Nonlinear Science and Numerical Simulation, vol. 18, no. 11, pp. 3061-3074, 2013.

[17] Y. Wang, L. Liu, and Y. Wu, "Positive solutions for a class of higher-order singular semipositone fractional differential systems with coupled integral boundary conditions and parameters," Advances in Difference Equations, vol. 2014, article 268, 2014.

[18] D. J. Guo and V. Lakshmikantham, Nonlinear Problems in Abstract Cones, vol. 5 of Notes and Reports in Mathematics in Science and Engineering, Academic Press, New York, NY, USA, 1988. 


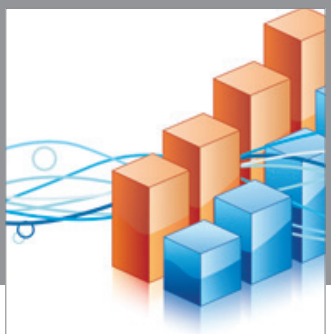

Advances in

Operations Research

vatem alat4

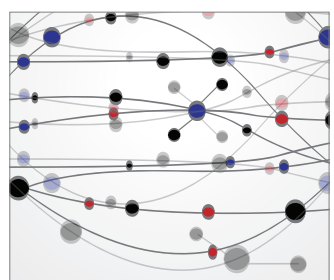

\section{The Scientific} World Journal
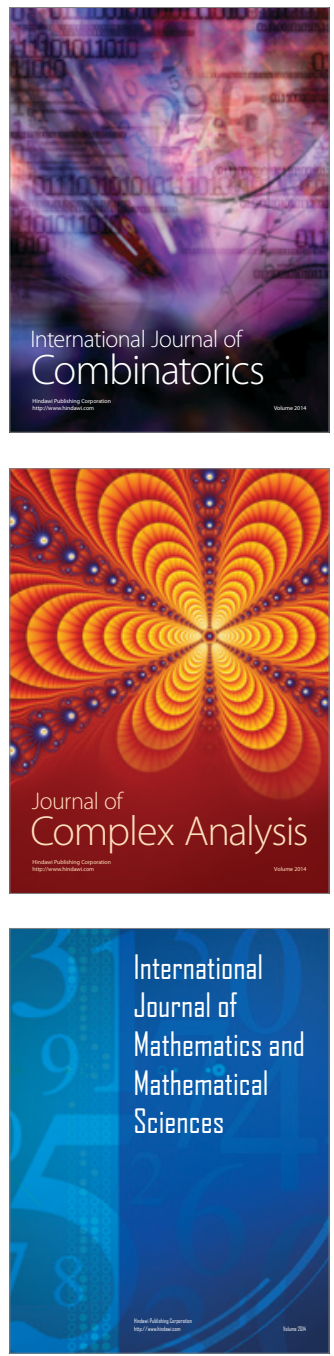
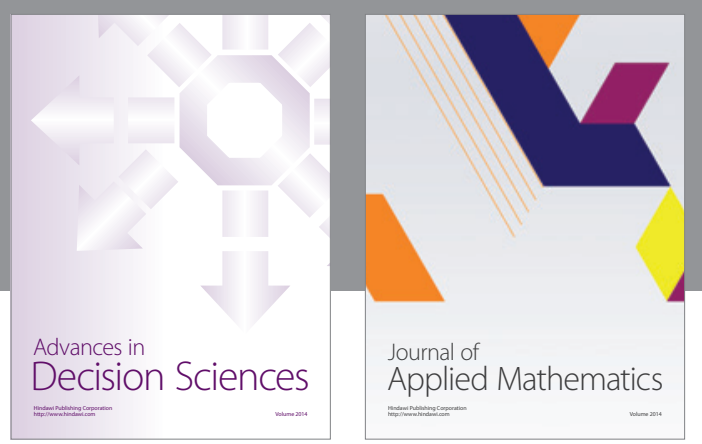

Algebra

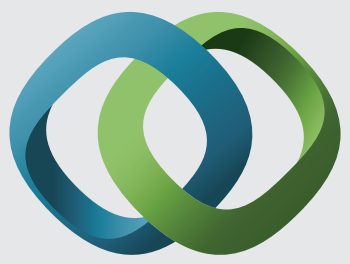

\section{Hindawi}

Submit your manuscripts at

http://www.hindawi.com
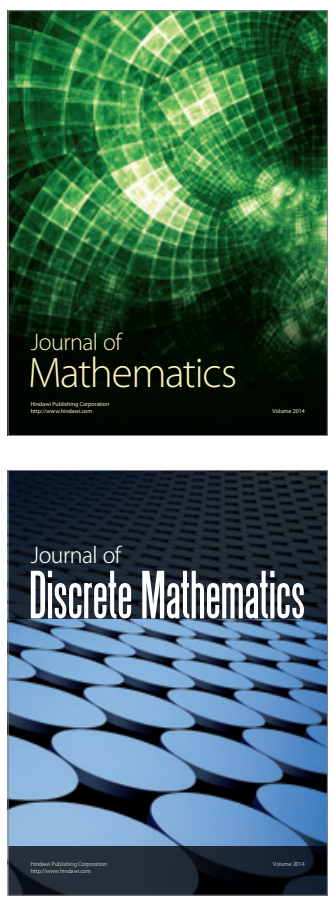

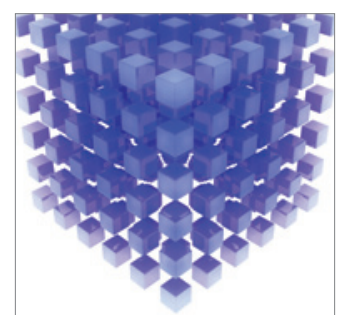

Mathematical Problems in Engineering
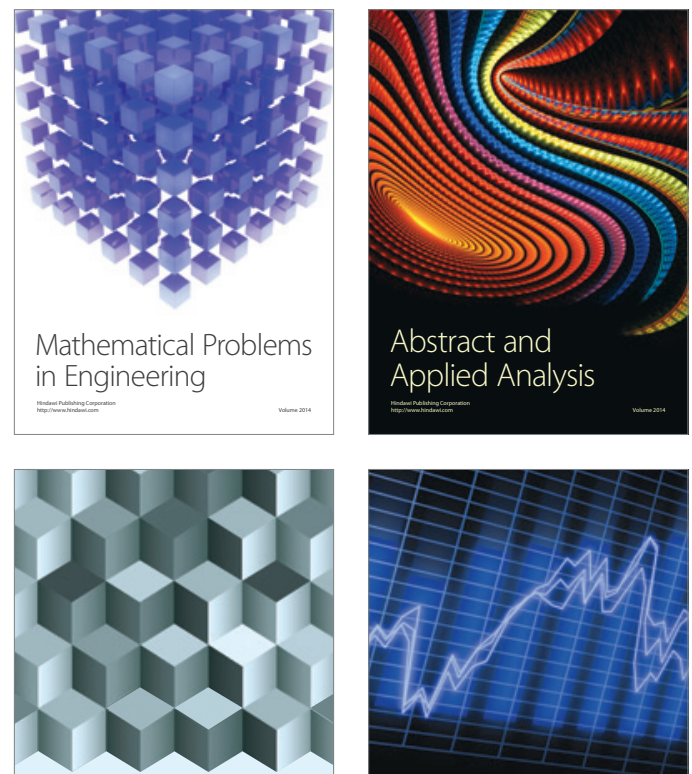

Journal of

Function Spaces

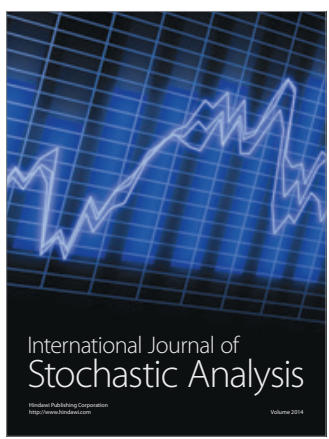

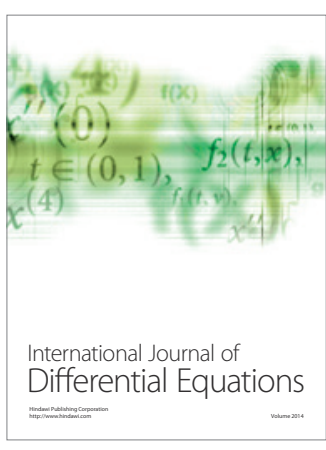
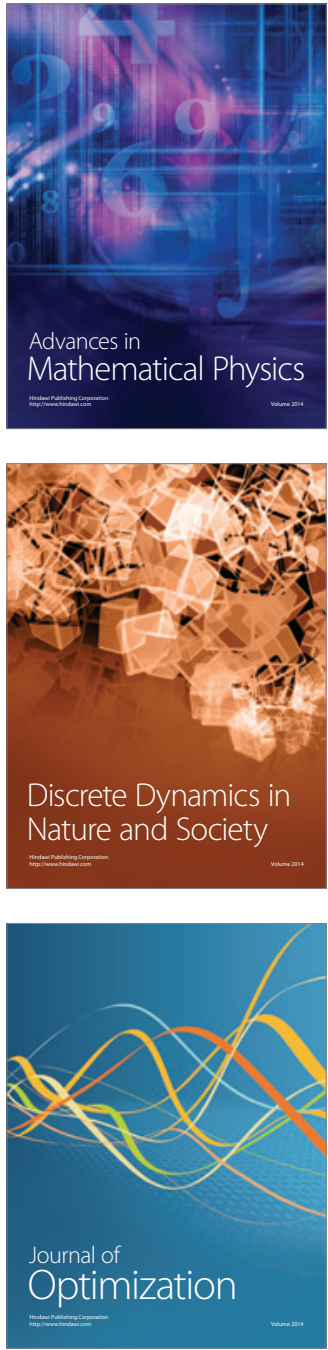